\title{
Ketamine: Ideal Pain Management for Traumatic Patients at High-Altitude
} Kyle Davis*

Advanced Tactical Practitioner, Boise State University, Boise, ID, USA

*Corresponding author: Kyle Davis, Advanced Tactical Practitioner, Boise State University, Boise, ID, USA, Tel: 7278936254; E-mail: kyleadavis5@yahoo.com Received date: June 07, 2017; Accepted date: June 19, 2017; Published date: June 26, 2017

Copyright: (c) 2017 Davis K. This is an open-access article distributed under the terms of the Creative Commons Attribution License, which permits unrestricted use, distribution and reproduction in any medium, provided the original author and source are credited.

\begin{abstract}
Altitude contributes to a wide variety of physiological changes within the body. Changes that disrupt the amount of oxygen delivered to the cells, altering the acid-base balance, resulting in abnormalities like skip breathing, hypercapnia and hypoxia. All issues that are uncommon breathing ambient air. As more and more adventurous thrillseeking individuals travel to high elevations, it becomes more paramount for medical providers to understand these effects. With special attention on the pharmacodynamics of drugs in a lower oxygen environment. Traumatic patients are of special interest at altitude because they require constant monitoring as well as proper resource management. Within every provider trauma protocol is pain management of the patient. Adverse side effects can always present from improper dosing of opioid narcotics, however lower partial pressures of $\mathrm{O}_{2}$ amplify these concerns. Concerns mainly associated with the depression of the respiratory drive. Thus, increasing the risk of administering narcotics at altitude. Ketamine can successfully accommodate the need for analgesia at altitude, while eliminating the serious potential for CNS depression. This article seeks to educate and promote the usage of ketamine in high-altitude traumatic patients. The end goal is the confidence needed to avoid errors of omission rather commission in any austere environment.
\end{abstract}

Keywords: Ketamine; Versed; Reemergence phenonmenon; Special operations medicine; Altitude medicine; Altitude pain management; Special amphibious reconnaissance corpsman

\section{Special K}

Identifying the appropriate analgesic treatment can often be an extremely controversial topic.

Controversial in the sense that no universal protocol is ever implemented across the board. Which isn't a cause for concern since the best approach isn't a universal one. Analgesia, a variable that is situational dependent, must be understood and accounted in any environment as a medical provider. However, the inherent effects at altitude will limit the selection of drugs and may call for implementation of a specific analgesia protocol.

The adverse effects of opioids are extremely fatal, toss in lower partial pressure of oxygen then that of the $21 \%$ existing in ambient air, altitude dosing becomes increasingly more difficult. Diligence when choosing the specific analgesic agent is imperative. Understanding the adverse effects, and pharmacology to include but not limited to onset, duration/half-life, and peak plasma time of agents used to achieve analgesia will aid in the confidence to administer at altitude [1].

Ketamine, without reservation is an excellent single dose agent for the high-altitude environment. Ketamine a dissociative hypnotic is ideal for the specific environment due to the absence of respiratory depression which at altitude can be potentiated with opioids like morphine, fentanyl, hydromorphone and meperidine. Ketamine however can be a difficult drug to dose due to its short duration of action in the body. Knowing the desired effect, you are trying to achieve initially is the most important factor. Trying to take the edge off a sprained anterior talofibular ligament will be different then establishing an airway via surgical cricothyroidotomy. Both which ketamine can complement effectively. In short, the mechanism of injury, injuries sustained, signs/symptoms, evacuation time and anticipating future interventions will all determine the specific type of ketamine dosing [2].

\section{P's}

Dosing of ketamine can be broken up into 3 distinct phases known as the 3 P's. The 3 P's define the range of sedation needed to achieve the desired effect. The first $\mathrm{P}$, the pain dose, is given for mild to moderate pain without fully sedating the patient and severely altering their mental status. The pain dose is ideal for patients who need to stay alert and oriented. Administration of ketamine intravenous/intraosseous at $0.1-0.5 \mathrm{mg} / \mathrm{kg}$ is within the safe zone for pain management. Intramuscular ketamine is effective and like all drugs will have a slower onset, however IM ketamine may be ideal for the pain zone and ambulatory patients. Most strains, sprains, and minor fractures are ideal for the pain zone of ketamine at altitude.

The 2nd $\mathrm{P}$ is the party/partial dissociation dose. This dose can pose problems in the field/altitude environment. When patients fall into this dosing regimen they tend to have a higher likelihood of experiencing re-emergence phenomenon. Re-emergence phenomenon unfortunately is one of the side effects of ketamine that can complicate/ potentiate the traumatic experience for the patient. It is characterized in battlefield medicine as a dream-like state with vivid hallucinations causing combative behavior of the patient $(3,205)$. Entering the party zone too often, more than once, will increase the likelihood of reemergence phenomenon which is problematic for the provider at altitude. Thus, patients should never be left in the party zone dosing of ketamine. No practical use comes from this area in terms of what would need to be done at altitude. The criteria for the party dose is $0.3-0.8 \mathrm{mg} / \mathrm{kg}$.

Be bold and confident in your drug administration; don't linger in the party zone. Adhering to the one and only rule of the party zone will 
simplify the patients care. If using an initial sedation bolus/bump it is wise to implement dual agents to better achieve all components of anesthesia.

Midazolam a benzodiazepine works exceptionally well with ketamine. Implementing a dual agent concoction with both ketamine and midazolam improves sedation, analgesia, as well as mitigating the possibility of reemergence delirium. Dosing with a $2 \mathrm{mg}$ bump IM/ slow IV push of midazolam post $200 \mathrm{mg}$ bump of ketamine corresponds well when performing tubal thoracotomy's, cricothyroidotomy's or any other lifesaving surgical intervention. Know and keep track of your dosing with benzos, the adverse effects will be amplified at altitude. Maintain increments of $25 \%$ the originally induction dose of midazolam if signs show that anesthetic effects are lightening.Other agents that can be considered with ketamine are diazepam, fentanyl (for breakthrough pain), and regional nerve blocks per procedure (lidocaine or bupivacaine).

The final $\mathrm{P}$ of ketamine dosing is the procedural sedation phase. Dosing roughly about $1.0-3.0 \mathrm{mg} / \mathrm{kg}$ to achieve the proper effect. Making the decision to sedate a patient to perform a life-saving procedure needs to be done quickly. Use the $200 \mathrm{mg}$ IV/IO bolus as a reference to achieve initial sedation. Titrate your dose to maintain procedural sedation. Keep in mind the factors involved in sedating your patient (i.e. airway, foley catheter, nasogastric tube etc.). Being confident in altitude physiology along with proper anesthesia MSMAID precautions, sedating at altitude will be less precarious. Ketamine does cover two areas of anesthesia, analgesia and amnesia. The third, muscle relaxation is not covered by ketamine, bringing forth the need to implement midazolam to fully achieve complete anesthesia [3].

\section{Ketamine Infusion}

Setting up a ketamine infusion can be extremely useful in prolonged field care scenarios. This allows the provider to focus his attention to different aspects of the patient, while maintain concurrent analgesia with ketamine. Mix $500 \mathrm{mg}$ of ketamine in a $500 \mathrm{ml}$ normal saline bag. This gives you a ratio of $1 \mathrm{mg} / \mathrm{ml}$. The maintenance dose throughout can be the patients weight in kilograms, which will equal to the number of drops per minute. The result is roughly $4 \mathrm{mg} / \mathrm{kg} / \mathrm{hr}$. Adjust to effect as needed if the patient becomes light at any point. Remember to always include your induction dose before setting your drip. $200 \mathrm{mg}$ $\mathrm{IM} / \mathrm{IV}$ is a good start.
Ketamine side effects aren't of big concern at altitude. Ketamine does not cause cardiovascular suppression. Although in certain trauma patients blood pressure support may be required if natural catecholamines are exhausted. This can be augmented with epinephrine and a whole blood transfusion [4]. Keep in mind that analgesia isn't your only concern with a multi system trauma patient. Brief and subtle increased intercranial pressure may result in the rapid infusion of ketamine in high doses. Of one combat casualty above $10,000 \mathrm{ft}$ in Northwest Africa, ketamine was giving IV rapidly at 100 $\mathrm{mg}$. The initial complaint of the patient before induced was an extreme pounding headache. How much fluid shift took place remains unknown. But at high-altitude with a patient suffering from HACE (High Altitude Cerebral Edema) it is good to be aware of what side effects can arise. Most of the fluid shift is a result of the ketamine blocking the NMDA (N-methyl-D-aspartate) receptor that affects the LTP (long term potentiation) in the CA1 region of the Hippocampus. This however is the trade-off of ketamine to deliver its dissociative effects [5].

Ketamine's wide range of usage in traumatic patients with little to no side effects that aggravate the inherent physiological changes at altitude makes it the ideal choice for pain management. Optimal resource management in austere environments is crucial. Ketamine being a dissociative hypnotic can account for you analgesic resource needs. Thus, making ketamine the ideal single agent for pain management at high-altitude.

\section{References}

1. Auerbach PS (2012) Wilderness medicine. (6th edn), Elsevier Mosby, Philadelphia.

2. USSOCOM Office of the Command Surgeon (2013) Advanced tactical paramedic protocols handbook. (7thedn), Breakaway Media, LLC, St Petersburg.

3. Callaway D (2016) Ketamine and pharmacology. Proceedings of the special operations medical association scientific assembly.

4. Farr WD, Fenton LH, Clayton R, Thompson GD, Allen R, et al. (2008) Special operations forces medical handbook. (2ndedn), US Government Printing Office, Washington DC.

5. Naval sea systems command (2011) US Navy dive manual. (Revision 6), US Government printing office, Washington DC. 